\title{
Weibel instability in anisotropically inhomogeneous plasmas
}

\author{
S. Tomita* and Y. Ohira
}

Department of Physics and Mathematics, Aoyama Gakuin University, Japan

E-mail: Eomisaradphys.aoyama.ac.jp, ohiradphys.aoyama.ac.jp

The Weibel instability is thought to be important for particle acceleration and generation of magnetic fields in relativistic shocks. However, the magnetic field produced by the Weibel instability cannot occupy large regions because of the rapid decay. Nonlinear evolution of the Weibel instability has been investigated in uniform plasmas or shocks propagating into uniform plasmas so far. In order to study nonlinear evolution of the Weibel instability in inhomogeneous background plasmas, we perform two-dimensional particle-in-cell simulations. We find that spatially anisotropic density structures produce anisotropic velocity distributions, so that the magnetic field fluctuations are excited by the Weibel instability. Relativistic shocks propagating into inhomogeneous plasmas are expected to produce such anisotropic density structures in the downstream region. Therefore, magnetic fields generated by the Weibel instability could occupy regions larger than previously thought. If so, the maximum energy of accelerated particles would become larger than previously thought.

The 34th International Cosmic Ray Conference,

30 July- 6 August, 2015

The Hague, The Netherlands

*Speaker. 


\section{Introduction}

In the high-energy astrophysical phenomena such as a pulsar wind nebulae, jets from active galactic nuclei and gamma-ray bursts (GRBs), a relativistic supersonic outflow is generated. The bulk kinetic energy of the flow is dissipated by a relativistic collisionless shock. For unmagnetized relativistic shocks, the Weibel instability [W] has a crucial role in the shock dissipation and particle acceleration [2], []]. According to observations of GRBs, magnetic fields are though to be strongly amplified in a large emission region of GRB [团], although some observations suggest no amplification of magnetic fields in GRB [[]]. The Weibel instability occurs in plasmas with anisotropic velocity distributions and amplifies magnetic field fluctuations. In fact, particle-in-cell (PIC) simulations showed the magnetic field amplification by the Weibel instability in the shock transition region. However, PIC simulation showed that the magnetic field produced by the Weibel instability cannot occupy large regions because of the rapid decay.

The Weibel instability has been investigated in uniform plasmas or shocks propagating in uniform plasmas. However, there must be density fluctuations in realistic situations. In this study, we investigate the nonlinear evolution of the Weibel instability in an inhomogeneous plasma by using two-dimensional PIC simulations. Especially, we consider an anisotropic density distribution as inhomogeneity, which is expected in the downstream region of relativistic shocks.

\section{Simulation setup}

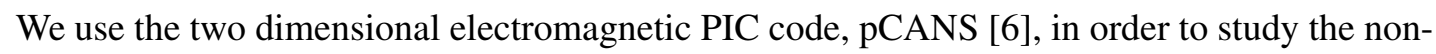
linear evolution of the Weibel instability in an inhomogeneous plasma. We set a two-dimensional simulation box in the xy plane with the periodic boundary condition in both directions. The simulation box size is $L_{\mathrm{x}}=L_{\mathrm{y}}=120 \mathrm{c} / \omega_{\mathrm{p}}$ where $\omega_{\mathrm{p}}$ and $c$ are the plasma frequency defined by the total number density of electron-positron plasmas and the speed of light, respectively. The cell size and time step are $\Delta x=\Delta y=0.1 c / \omega_{\mathrm{p}}$ and $\Delta t=0.05 \omega_{\mathrm{p}}^{-1}$, respectively. Initially, we prepare two counter-streaming unmagnetized electron-positron plasmas with the drift velocities, $v_{\mathrm{d}}= \pm 0.5 c$, where the counter-streaming direction is the $\mathrm{x}$ direction. Each electron-positron plasma has the same density and thermal velocity, $v_{\text {th }}=0.1 c$.

For a spatial distribution of the electron-positron plasma, we consider two cases in this study:

1. $n(x, y)=n_{0}$

2. $n(x, y)=n_{0}\left\{1+0.5 \sin \left(2 \pi y / L_{\mathrm{y}}\right)\right\}$

One is the homogeneous distribution and the other is the inhomogeneous and spatially anisotropic distribution.

\section{Simulation results}

In Fig. 1, we show the time evolution of the mean energy density of magnetic fields in the simulation box. The black and red curves show the energy density for the homogeneous and inhomogeneous distributions, respectively. For the homogeneous distribution (black curve), initially, 


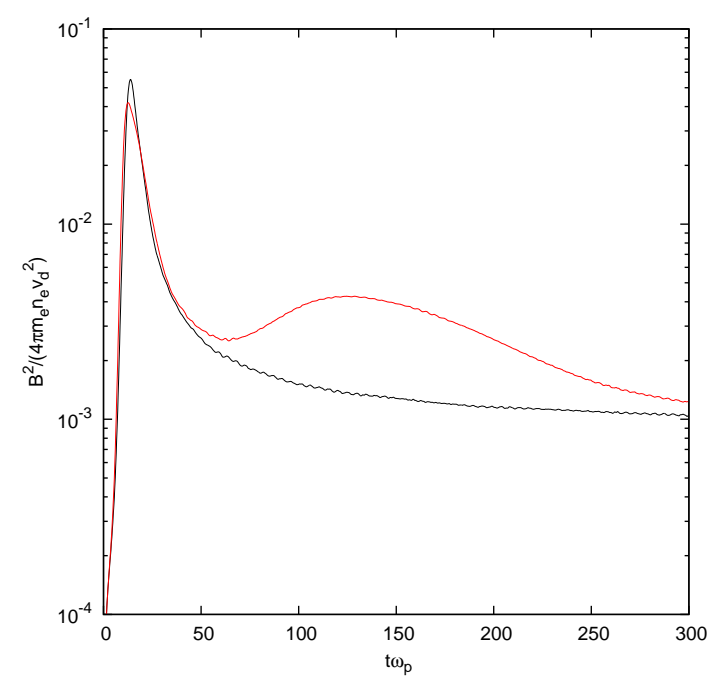

Figure 1: Time evolution of the mean energy density of magnetic fields for the homogeneous (black) and inhomogeneous (red) distributions. The energy density is normalized by the initial mean kinetic energy density.

the magnetic field grows exponentially as predicted by the linear analysis of the Weibel instability. After the saturation at $t \approx 13 \omega_{\mathrm{p}}^{-1}$, the magnetic field simply decays. On the other hand, for the inhomogeneous distribution (red curve), after the first saturation at $t \approx 13 \omega_{\mathrm{p}}^{-1}$, the magnetic field starts to grow again from $t \approx 60 \omega_{\mathrm{p}}^{-1}$. After the second saturation at $t \approx 120 \omega_{\mathrm{p}}^{-1}$, the magnetic field decays slowly. Therefore, interestingly, the magnetic field is amplified for longer time in the inhomogeneous distribution even though the total kinetic energy is the same as that of the homogeneous distribution. The origin of the second growth of the magnetic field is discussed in the next section.
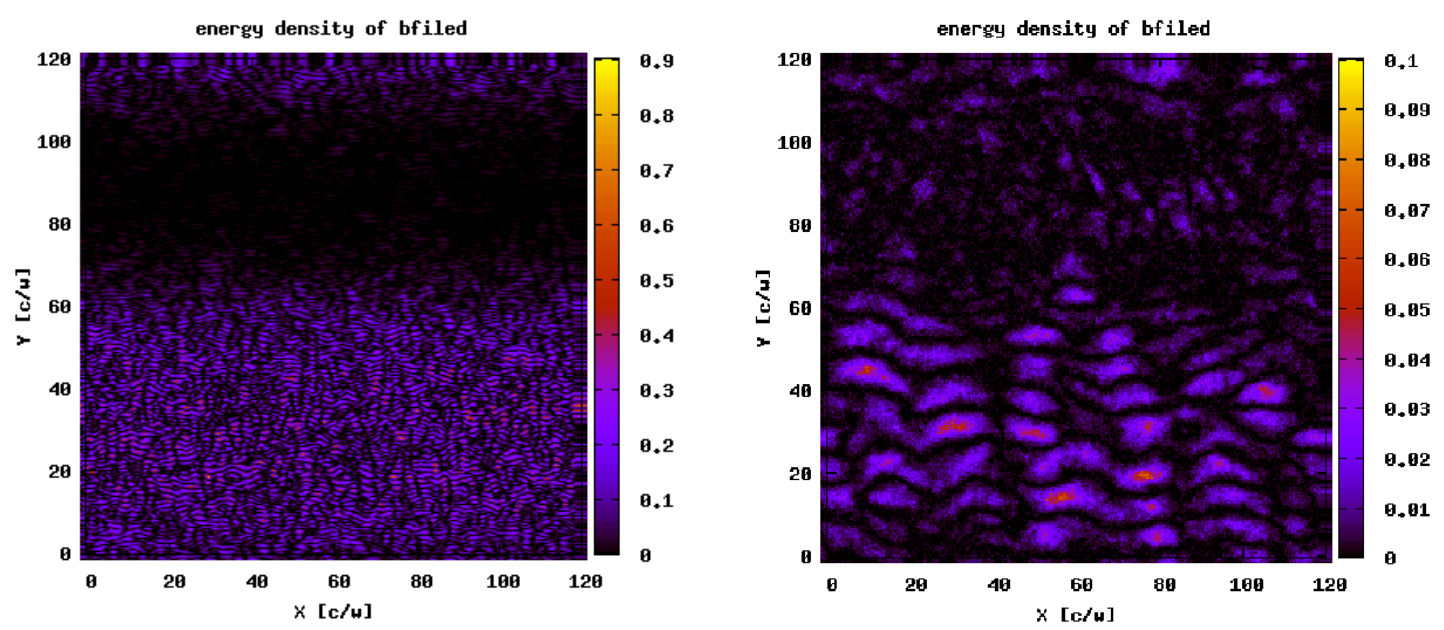

Figure 2: Magnetic field energy density at the first (left) and second (right) saturations for the inhomogeneous distribution. 
In Fig. 2, we show spatial distributions of the energy density of magnetic fields at the time of the first (left panel) and second (right panel) saturations for the inhomogeneous distribution. At the time of the first saturation (left panel), $t=13 \omega_{\mathrm{p}}^{-1}$, magnetic fields are strongly amplified in the high density region $\left(y \approx 30 c / \omega_{\mathrm{p}}\right)$ compered with the low density region $\left(y \approx 90 c / \omega_{\mathrm{p}}\right)$. This is because the growth rate of the Weibel instability is given by $\gamma \propto \omega_{\mathrm{p}}$ and increases with the density. As time goes on, magnetic fields grow in the low density region, but the amplitude of the magnetic field fluctuation is smaller than that in the high density region because the initial kinetic energy density is small in the low density region. Around the time of the first saturation, the typical length scale of the magnetic field fluctuation is about the skin depth, $c / \omega_{\mathrm{p}}$. At the time of the second saturation (right panel), $t=120 \omega_{\mathrm{p}}^{-1}$, magnetic fields are amplified in the high density region and the length scale of the magnetic field fluctuation becomes about $20 \mathrm{c} / \omega_{\mathrm{p}}$.
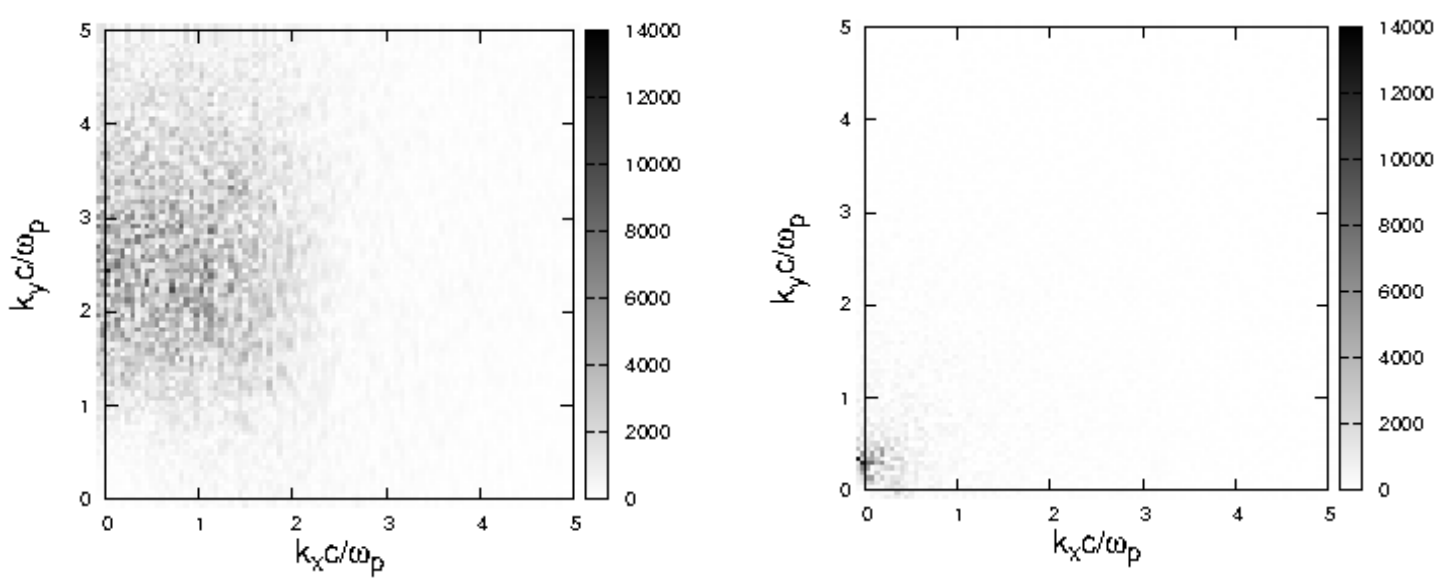

Figure 3: Fourier power spectrum of the z-component of magnetic fields at $t=13 \omega_{\mathrm{p}}^{-1}$ (left) and $t=$ $120 \omega_{\mathrm{p}}^{-1}$ (right) for the inhomogeneous distribution.

In order to see the evolution of the length scale more clearly, in Fig. 3, we show the Fourier power spectrum of the z-component of magnetic fields, $B_{\mathrm{z}}$. The Fourier power spectra of $B_{\mathrm{Z}}$ at $t=13 \omega_{\mathrm{p}}^{-1}$ (first saturation) and $t=120 \omega_{\mathrm{p}}^{-1}$ (second saturation) are shown in the left and right panels of Fig. 3, respectively. As predicted by the linear analysis, magnetic field fluctuations are excited in the region, $k_{\mathrm{x}} \gtrsim 1 \omega_{\mathrm{p}} / c$ and $k_{y} \lesssim 1 \omega_{\mathrm{p}} / c$ at $t=13 \omega_{\mathrm{p}}^{-1}$. On the other hand, the peak is located around $\left(k_{\mathrm{x}}, k_{\mathrm{y}}\right)=\left(0,0.3 \omega_{\mathrm{p}} / c\right)$ at $t=120 \omega_{\mathrm{p}}^{-1}$. Therefor, we confirm that magnetic field fluctuations with larger length scale are excited by the second growth.

\section{Origin of the second growth of magnetic fields}

In Fig. 1, we have shown that magnetic field fluctuations start to grow after the saturation of the first Weibel instability. In this section, we discuss the origin of the second growth of magnetic field fluctuations. In this simulation, there is initially no magnetic field, so that the second growth of magnetic field is also expected to be due to the Weibel instability. To confirm that, in Fig. 4, we show the velocity distribution in the high density region where magnetic fields grow after the saturation of the first Weibel instability, $20 \mathrm{c} / \omega_{\mathrm{p}} \leq y \leq 40 \mathrm{c} / \omega_{\mathrm{p}}$, at $t=100 \omega_{\mathrm{p}}^{-1}$. One can see the 


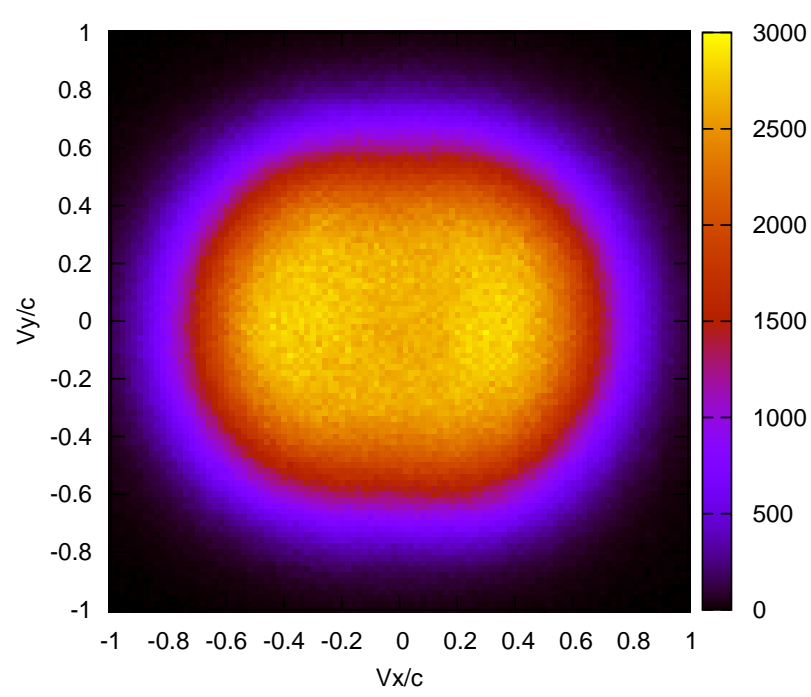

Figure 4: Velocity distribution in the high density region $\left(20 \mathrm{c} / \omega_{\mathrm{p}} \leq y \leq 40 \mathrm{c} / \omega_{\mathrm{p}}\right)$ at $t=100 \omega_{\mathrm{p}}^{-1}$ for the inhomogeneous distribution.

anisotropic velocity distribution in Fig. 4. The velocity dispersion in the x-direction is larger than that in the $y$-direction and the temperature anisotropy is $A=T_{\mathrm{x}, \mathrm{z}} / T_{\mathrm{y}}-1 \approx 0.2$. According to the linear analysis [四], the dispersion relation for the anisotropic temperature plasma is given by

$$
0=\omega^{2}-k_{\mathrm{y}}^{2} c^{2}+\omega_{\mathrm{p}}^{2}\left\{A-(A+1) \frac{\omega}{v_{\text {th }, \mathrm{y}} k_{\mathrm{y}}}\right\} Z\left(\frac{\omega}{v_{\text {th }, \mathrm{y}} k_{\mathrm{y}}}\right),
$$

where $v_{\text {th,y }}$ and $Z$ are the velocity dispersion in the y-direction and the plasma dispersion function, respectively, and the wave vector is assumed to be $\vec{k}=k_{\mathrm{y}} \vec{e}_{\mathrm{y}}$. For a small anisotropy $(A \ll 1)$, the maximum growth rate is given by [प]

$$
\gamma_{\max }=\sqrt{\frac{4}{27 \pi}} \frac{A^{3 / 2}}{1+A}\left(\frac{v_{\text {th,y }}}{c}\right) \omega_{\mathrm{p}} \text { for } k_{\mathrm{y}}=\sqrt{\frac{A}{3}} \frac{\omega_{\mathrm{p}}}{c}
$$

For $A \approx 0.2$ and $v_{\text {th }, \mathrm{y}} \approx c$, the mode with $k_{\mathrm{y}} \approx 0.26 \omega_{\mathrm{p}} / c$ has the maximum growth rate, $\gamma_{\max } \approx$ $1.6 \times 10^{-2} \omega_{\mathrm{p}}$, which are consistent with our simulation results.

The temperature anisotropy originates from the anisotropic density structure. After the first saturation of the Weibel instability, the velocity dispersion of plasma becomes large ( $v_{\text {th }} \approx c$ for this simulation) and high velocity particles start to escape from the high density region to the low density region. For the density structure that we use in this study, particles with a large $v_{\mathrm{y}}$ escape from the high density region. As a result, the temperature in the y-direction becomes smaller than that in the $\mathrm{x}$ and $\mathrm{z}$-direction. 
Therefore, we conclude that the second growth of magnetic fields is due to the Weibel instability and the temperature anisotropy after the first saturation is produced by the anisotropic density structures.

\section{Discussion}

In sections 3 and 4, we showed that anisotropic density structures in collisionless plasmas produce anisotropic velocity distributions and amplify magnetic fields by the Weibel instability. Such a situation can be expected in the downstream region of relativistic shocks because upstream isotropic density structures are compressed very anisotropically in the shock downstream region. Therefore, thanks to this mechanism, the magnetic field produced by the Weibel instability could occupy large downstream regions of relativistic collisionless shocks propagating into inhomogeneous plasmas. If so, the second Weibel instability that we observed in this study could be important for radiation from GRBs [[]], particles accelerations in the Weibel mediated shocks [3] and generation of magnetic fields $[\mathbb{Q}, \mathbf{Q}, \mathbf{0}, \mathbf{0}]$. Furthermore, the Weibel instability is expected to have an important role even for nonrelativistic shocks [], [2] and shocks produced in leaser experiments [[13, [4]].

\section{Acknowledgments}

We thank R. Yamazaki, J. Shimoda, Y. Shoji, M. Nogami, E. Kobayashi for useful comments. We also thank ISSI (Bern) for support of the team "Physics of the injection of particle acceleration at astrophysical, heliospheric, and laboratory collisionless shocks". The software used in this work was in part developed in pCANS at Chiba University. Numerical computations were carried out on Cray XC30 at Center for Computational Astrophysics, National Astronomical Observatory of Japan. ST is supported by the Komoda fellowship.

\section{References}

[1] E. S. Weibel, Particle Acceleration in Relativistic Collisionless Shocks: Fermi Process at Last?, PRL, 2, 83-84, 1959.

[2] M. Medvedev and A. Loeb, Generation of Magnetic Fields in the Relativistic Shock of Gamma-ray Burst Sources, ApJ, 526, 697-706, 1999.

[3] A. Spitkovsky, Particle Acceleration in Relativistic Collisionless Shocks: Fermi Process at Last?, ApJ, 682, L5-L9, 2008.

[4] E. Waxman, Gamma-Ray-Burst Afterglow: Supporting the Cosmological Fireball Model, Constraining Parameters, and Making Predictions, ApJ, 485, L5-L8, 1997.

[5] P. Kumar and R. B. Duran, On the Generation of High-Energy Photons Detected by the Fermi Satellite from Gamma-Ray Bursts, MNRAS, 400, L75-L79, 2009.

[6] pCANS, http://www.astro.phys.s.chiba-u.ac.jp/pcans/

[7] R. C. Davidson, D. A. Hammer, I. Haber, and C. E. Wagner, Particle Acceleration in Relativistic Collisionless Shocks: Fermi Process at Last?, PRL, 2, 83-84, 1959.

[8] R. Schlickeiser and P. K. Shukla, Cosmological Magnetic Field Generation by the Weibel Instability, ApJ, 599, L57-L60, 2003. 
[9] Y. Fujita and T. Kato, A Possible Origin of Magnetic Fields in Galaxies and Clusters: Strong Magnetic Fields at z 10?, MNRAS, 364, 247-252, 2005.

[10] M. Medvedev, L. O. Silva, and M. Kamionkowski, Cluster Magnetic Fields From Large-Scale Structure and Galaxy Cluster shocks, ApJ, 642, L1-L4, 2005.

[11] T. Kato, Nonrelativistic Collisionless Shock in Weakly Magnetized Electron-ion Plasmas: Two-Dimensional Particle-In-Cell Simulation of Perpendicular Shock, ApJ, 721, 828-842, 2010.

[12] Y. Matsumoto, T. Amano, T. N. Kato, and M. Hoshino, Stochastic Electron Acceleration During Spontaneous Turbulent Reconnection in A Strong Shock Wave, Science, 347, 974-978, 2015.

[13] W. Fox, G. Fiksel, A. Bhattacharjee, P.-Y. Chang, K. Germaschewski, S. X. Hu, and P. M. Nilson, Filamentation Instability of Counterstreaming Laser-Driven Plasmas, PRL, 111, 225002, 2013.

[14] C. M. Huntington et al., Observation of Magnetic Field Generation via the Weibel Instability in Interpenetrating Plasma Flows, Nature Physics, 11, 173-176, 2015. 